\section{Nutrition Specific Interventions for Management of Malnutrition in Eastern Equatoria state, South Sudan - Challenges and Lessons for Non-Governmental Organizations}

\section{Abstract}

Background: Decades long efforts have been exerted by Non-Governmental Organizations in partnership and collaboration with Governments, UN agencies and Donor communities to address malnutrition through nutrition specific interventions in resource poor and conflict prone countries. The impact of nutrition specific interventions cannot be underscored. In this paper, we argue that whereas nutrition specific responses do tackle under nutrition among vulnerable populations, they often tend to be limited in scope and sustainability.

Method: This was a cross-sectional study that employed qualitative techniques for data collection. Primary data was obtained from purposively selected NGOs $(n=17)$ that implement nutrition programs in Eastern Equatoria state through key informant interviews. This was supplemented by secondary data from existing literature. Data was summarized, triangulated and analyzed for contents to generate the themes in line with study objectives.

Results: This study has shown that although nutrition specific responses play a vital role in tackling malnutrition, they often tend to be limited in scope. Tackling all the root causal factors of malnutrition remains a challenge. Such challenges often resulted into relapse despite reported high proportion (at least $75 \%$ ) of children in treatment programs being discharged as cured.

Conclusion: In a short run, nutrition specific interventions can be effective in saving lives of malnourished individuals. However, there is need to ensure that both nutrition specific and sensitive interventions are given proportional investment priorities. This can be done by allocating adequate resources to both interventions in addition to building resilience of communities to local shocks. Otherwise, nutrition specific interventions will continue for many more years to come.

Keywords: Malnutrition; Nutrition specific interventions; Challenges; Lessons; South Sudan; Non-Governmental organizations
Amegovu AK ${ }^{1 *}$, Mori J'2, Chewere $\mathrm{T}^{3}$, Muyima $\mathrm{L}^{4}$, Jokudu $\mathrm{S}^{5}$ and Mawadri $\mathbf{M}^{3}$

\author{
1 Department of Food Science and \\ Technology, University of Juba P.O. 82 \\ Juba, South Sudan \\ 2 UNICEF, South Sudan \\ 3 Andre Foods International (AFI) P.O. Box \\ 830 Entebbe, Uganda \\ 4,5 Andre Foods South Sudan (AFSS), South \\ Sudan
}

*Corresponding author:

Andrew Kiri Amegovu

” kiri_andrew@yahoo.com

Department of Food Science and Technology, University of Juba, Juba, South Sudan.

Tel: +211915 596994

Citation: Amegovu AK, Mori J, Chewere T, Muyima L, Jokudu S, et al. (2020) Nutrition Specific Interventions for Management of Malnutrition in Eastern Equatoria state, South Sudan - Challenges and Lessons for Non-Governmental Organizations. J Clin Nutr Diet. Vol 6 No.1:1

\section{Introduction}

Nutrition is essential for a population and particularly children to survive grow and achieve their full potential in terms of social, economic, intellectual and physical. The United Nations identifies adequate nutrition as a critical driver for progress in other Sustainable Development Goal (SDG) indicators like health, education, employment, empowerment of women as well as reduction of poverty and inequality. While the importance of nutrition for development is universally recognized, global efforts in combating hunger and malnutrition have had mixed results [1-3]. According to International Food Policy Research Institute (IFPRI), the prevalence of stunting among children under five 
years declined to 23.8 percent from 36.9 percent between 1990 and 2015 However, in 2017, the Food and Agricultural Organization of the United Nations (FAO) indicated that number of undernourished people increased from 777 million to 815 million between 2015 and in 2016, a total of about 155 million children under age 5 were too short for their age, approximately 52 million didn't weigh enough for their height and another close to 41 million were overweight Malnutrition is linked to nearly half of all deaths among children under the age of five [4-7].

Under nutrition is the most common form of malnutrition in the developing countries. Of the 233 million undernourished people in Africa, 220 million are from the Sub Saharan. While South Sudan is deficient of globally comparable data, estimates show that the food and nutritional shortfalls are dire. For example, by January-March 2019, 5.2 million South Sudanese (49\% of the total population) continued to face acute food insecurity $[7,8]$. The weighted prevalence of acute malnutrition in the 5 states stood at $13.3 \%$ and $2.8 \%$ for GAM (General Acute Malnutrition) and Severe Acute Malnutrition (SAM) respectively.

In South Sudan, livelihoods have been disrupted by the recurrent conflict and resulting insecurity. The protracted economic crisis is characterized by hyperinflation and depreciation of the South Sudanese pound (SSP), soaring food prices, a high cereal crop deficit and drought in parts of the country as contributors to the high level of food insecurity and malnutrition Despite the implementation of the different nutrition programmes trends shown in (Figure 1) according to the Food Security and Nutrition Monitoring Survey reports [9], the prevalence of acute malnutrition among children $<5$ years remains at serious to alert level of 10 percent below the emergency threshold of 15\% (WHO threshold).

At the immediate level, the causes of malnutrition include inadequate dietary intake and ill health primarily from repeated infectious diseases. However, these immediate causes are exacerbated by many factors, notably conflicts characterized by political instability; drought and disasters linked to climate change, population growth and rising food prices [10].

In order to address this challenge, nutrition specific programs have been adopted as an intervention strategy by both government and Non-Governmental Organizations. These programs address immediate and some underlying causes of malnutrition. Specific interventions that address immediate causes of malnutrition include treatment of Severe Acute Malnutrition (SAM) through Inpatient Therapeutic Programme (ITP) and Outpatient Therapeutic care Programme (OTP), treatment of Moderate Acute Malnutrition (MAM) through Targeted Supplementary Feeding programme (TSFP), disease management and maternal and child micronutrient supplementation. While those that address intermediate or underlying causes may include optimal infant and young child feeding practices (exclusive and complementary feeding with continued breastfeeding, minimum dietary diversity, responsive feeding), appropriate health care as well as adequate hygiene and sanitation. Most of these programs are targeted at children under the age of five, pregnant and lactating mothers as well as chronically ill patients and may be administered through health facilities, community-based care or both.
This paper therefore provides an insight into the nature of nutrition specific programs being implemented in South Sudan, establishes the challenges of implementing such programs and documents best practices by drawing lessons from practical experiences. Unlike previous literature, we hinge on the perspective of NGOs who have been working at the frontline of South Sudan's nutrition response in the face of the protracted conflict. It provides a good case to learn from nutrition specific interventions and improve overall nutrition programming in conflict situations especially in the context of developing countries (Table 1).

\section{Methods}

In order to provide an NGO perspective to nutrition specific interventions in South Sudan, data was collected from a crosssection of NGOs that are currently implementing nutrition programs in Eastern Equatoria state. This state of South Sudan was purposively selected because it is prone to hunger, food insecurity and high poverty levels. Within this region, prevalence of acute malnutrition is estimated at $12.2 \%$ which is in the Alert phase based on the WHO standards. The region is also characterized by insufficient availability of food, very poor quality and diversity of food (Minimum Acceptable Diet of $<5 \%$ and Minimum dietary diversity of $<15 \%$ ) relatively high prevalence of diseases and poor child care practices. Additionally, reduced access to food, nutrition and health services are linked to conflict including inter communal conflict in some counties.

The study used both primary data and secondary data. Primary data was obtained from select NGOs $(n=17)$ that implement nutrition

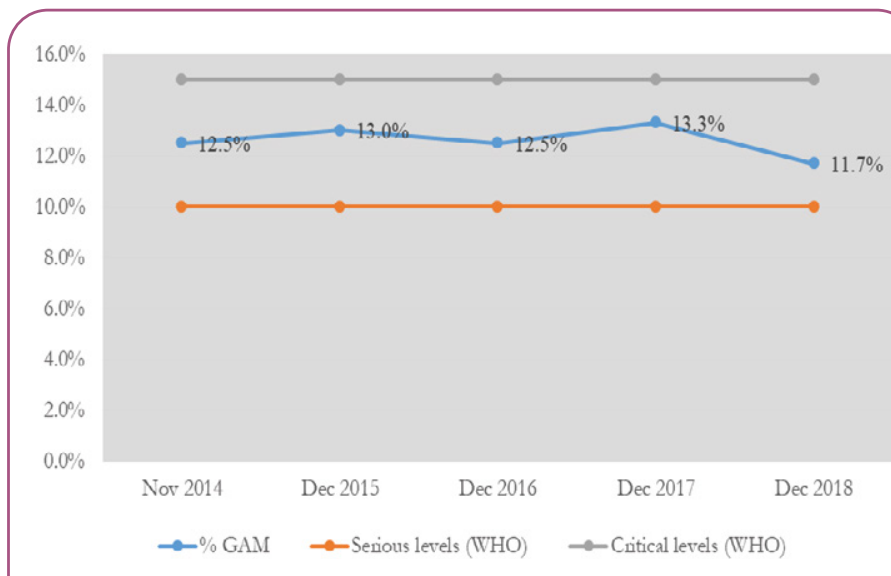

Figure 1 Trend in prevalence of acute malnutrition among children (6-59 months) from (2014-2018).

Table 1 Trend in prevalence of acute malnutrition among children (6-59 months (2014-2018) in South Sudan (UNICEF).

\begin{tabular}{|c|c|c|c|}
\hline Month/Year & \% GAM & $\begin{array}{c}\text { Serious levels } \\
\text { (WHO) }\end{array}$ & $\begin{array}{c}\text { Critical levels } \\
\text { (WHO) }\end{array}$ \\
\hline Nov-14 & $12.50 \%$ & $10 \%$ & $15 \%$ \\
\hline Dec-15 & $13.00 \%$ & $10 \%$ & $15 \%$ \\
\hline Dec-16 & $12.50 \%$ & $10 \%$ & $15 \%$ \\
\hline Dec-17 & $13.30 \%$ & $10 \%$ & $15 \%$ \\
\hline Dec-18 & $11.70 \%$ & $10 \%$ & $15 \%$ \\
\hline
\end{tabular}


programs in Eastern Equatoria through key informant interviews. This was supplemented by secondary data from existing literature including nutrition and food security assessments, programme monitoring, progress and implementation reports, evaluation studies and academic publications on nutrition and food security in the areas as well as other related contexts where useful lessons could be drawn to inform the Eastern Equatoria case. The study also reviewed secondary data on nutrition specific programs implemented in the Equatorial region between 2009 and 2018.

Using a predesigned semi structured question guide, KIls were administered to the technical and management personnel involved in the implementation of nutrition programs within the select NGOs. During the KIIs, information was collected on the profile and experience of identified respondents after which a detailed discussion was held to provide an in-depth understanding of the nutrition practitioner's knowledge, practices, attitudes, experiences and overall views on effectiveness and challenges of nutrition specific programming. Secondary data was obtained through online resources e.g. academic libraries and UN/NGO websites and complemented with offline resources like program implementation reports and evaluation studies where access to such information was granted by the NGOs included in this study.

Information on profile and experience was coded and quantitatively summarized in a Microsoft Excel spreadsheet specifically designed for the survey and then exported to the Stata version 14 for cleaning and analysis. On the other hand, qualitative data was coded and a template developed through which emerging themes and associations were reviewed summarized, triangulated and analyzed.

\section{Results and Discussion}

\section{Characterization of respondents}

The key informants were drawn from various non-government organizations implementing nutrition specific programs in Eastern Equatoria state in South Sudan. A total of 17 key informants were recruited for this study. The respondents constituted nutrition officers (29\%) programme managers (23\%), enrolled nurses $(12 \%)$ and nutrition field officers (12\%). The respondents had between 7 to 60 months working experiences with the majority having implemented nutrition programs for at least 48 months. In terms of technical knowledge, most respondents had either a nutritional, public health or medical professional backgroundwhich coupled with relevant experience made them competent to undertake nutrition programming. Table 2 provides a summary of the characteristics of respondents.

\section{What nutrition specific programs do NGOs implement in Eastern Equatoria?}

The nutrition specific programmes implemented in Eastern Equatoria included; the Outpatient Therapeutic Programme (OTP) and Inpatient Therapeutic Programme (ITP) supported by UNICEF for treatment of Severe Acute Malnutrition (SAM) without medical complication and SAM with medical complication respectively. In addition to the Targeted Supplementary Feeding Program (TSFP) and Blanketed Supplementary Feeding Programme (BSFP) supported by UNWFP for the treatment of Moderate Acute Malnutrition (MAM). Andre Foods South Sudan (AFSS) among other UNWFP/UNICEF nutrition partners in Eastern Equatoria implements OTP and TSFP in Kapoeta East County.

These nutrition specific programmes were implemented by UNICEF and UNWFP nutrition partners through health facility $(17 \%)$, community-based care (5\%), and through both health facility and community-based care (78\% respectively). This finding corroborates with some of the most commonly used nutrition specific protocols for managing malnutrition which include 1) take-home ration of Ready to Use Therapeutic food (RUTF) through OTPs for treatment of SAM without medical complications, 2) ITP management of SAM with complications and 3) outpatient supplementary feeding comprised of takehome ration of $\mathrm{CSB}++$ for PLW and a RUSF for children $<5$ years (WFP/UNICEF/MOH Guideline). Studies have also shown that nutrition interventions provide cost savings for hospitalized patients (Buitrago, 2019).

Respondents elaborated that no specific approach is preferred because both home-based care and facility-based treatment offer peculiar advantages. Accordingly, at the facility, patients receive compressive treatment (including for other medical complications) owing to availability of requisite health infrastructure, supplies and technical personnel. On the other hand, community-based care increases coverage, complies with guidelines for treatment of MAM and can be suited for villages that don't have a nearby health facility and/or limited infrastructure. In some cases, respondents simply implemented an approach based on donor funding guidelines. They however agree that reduction in mortality and morbidity among children less than 5 years was significant only when health nutrition service at facility were combined with interventions at community level.

Findings further revealed that more than $70 \%$ of nutrition programming in Eastern Equatoria targeted children under 5 years of age (38\%) as well as pregnant and breastfeeding mothers (35\%). Another group of interest was people with diseases like tuberculosis and HIV/AIDs. Programs aimed at the entire population and the elderly were only $10 \%$ and $4 \%$ respectively. Existing literature supports prioritizing the groups mentioned above based on their vulnerability. Some of the reasons proposed for this targeting include to prevent morbidity and mortality of children due to malnutrition, stimulate proper growth (both inter uterine and after birth), increase adherence to treatment and cure rate among people with chronic illnesses (especially TB patients) and provide an opportunity for children to benefit from the first 1000 days of life [11-13].

When the respondents were asked what achievements, the programs that they implemented had registered, about $63 \%$ acknowledged that most of the clients that were enrolled into the different treatment programs recovered and were discharged as cured. $15 \%$ said they have registered positive change in behavior and attributed these positive changes to nutrition and health behavior change programming components received by their clients. Additionally, $11 \%$ said the capacity of the health workers and the community nutrition volunteers to 
manage acute malnutrition has improved during the course of the implementation of the said programs. In this regard they believed that the nutrition programs have registered some achievements. Other achievements highlighted include capacity building of national staff, improved documentation of nutrition data at community and organization levels which is used to track nutrition programming. Previous studies have reported positive gains from nutrition specific interventions. Such achievements include improved knowledge in nutrition and health care practices - particularly with regard to IYCF practices, hygiene and health care among mothers [14-18].

Nutrition Specific programming yielded commendable results in reducing malnutrition among children said one respondent and greatly reduced child morbidity and mortality.

"After 21 days of treatment with RUTF in the OTP, the child with $S A M$ without medical complications improved and gained weight and MUAC".

Inasmuch as there is reported decline in acute malnutrition trends among children <5years, there remains a critical gap in implementation of nutrition specific programs especially in addressing the underlying and basic factors of malnutrition within the resource poor South Sudan engulfed with inadequate infrastructure, insecurity, economic meltdown and negative socio-cultural dynamics leave alone very limited investment scope for sustainability of nutrition treatment programs. Such challenges often resulted into relapse despite reported high proportion (at least $75 \%$ ) of children in treatment programs being discharged cured. Also, the treatment protocols based on CMAM/IMAM guidelines used by majority of NGOs is silent or not very specific on how to sustain the nutritional wellbeing of the $75 \%$ discharged cured. The unanswered question remains the long-term sustainability of nutrition specific interventions rather than the blame game on NGOs that treatment programs does not work and its total waste. This is very wrong argument because NGOs are like doctors who treat malaria in sick persons using prescribed drugs scientifically proven to kill malaria parasites and after patient's recovery from malaria, re-infection (or relapse) could re-occur if there are no adequate preventive measures and this doesn't mean the treatment didn't work at all. If these types of malfunctions and misconception in managing malnutrition are not well addressed, the UN agencies, Donors, Governments and NGOs should brace for long term undertakings to manage malnutrition in the way it is being managed.

Although some achievements were made using the current approaches of management of malnutrition in Eastern Equatoria, respondents were not confident about sustainability of outcomes. Fears were raised about frequent stock-outs of essential supplies, heavy reliance on donor funding which affects program longevity and the narrow focus on treatment of acute malnutrition without any complementary programs to support prevention and homebased management of mild malnutrition besides lively programs which to them have either no real impact or have very minimal short term impact.

Positive outcomes were also realized only when the beneficiaries exhibited strict adherence to prescribed dosages and other utilization instructions. Using a randomized control trial, Olney et al. also reported no significant improvement in child nutritional outcomes after intervention period, which they partly attributed to the short-lived duration of programming. Where exit occurred before the target effectively recovered, benefits were shortlived. While majority thought that nutrition specific interventions were unsustainable, some were optimistic based on the heavy involvement of ministry of health in the CMAM component, with the hope that government would take over financing in the event of donor exit.

\section{Challenges of implementing nutrition specific programmes}

The challenges NGOs face in implementing nutrition specific programmes in Eastern Equatoria can be broadly categorized as operational and programmatic.

\section{a) Operational challenges}

These included all setbacks related planning, implementing and controlling resources allocated for nutrition programs. Key informants blamed the rampant operational challenges in the study area for short falls. The most prominent challenges identified ranged from poor infrastructure (including roads, shelters in health facilities and storage facilities for supplies) $(34 \%)$, insecurity (32\%) and limited capacity of health workers and the Community Nutrition Volunteers (CNVs) to implement the nutrition programmes (Figure 2).

Availability of requisite infrastructure in central to effectiveness of programme delivery both within and outside nutrition interventions. Poor infrastructure interrupts delivery nutrition services, effective monitoring and supervision of beneficiaries, limits spatial coverage and in some instances renders it impossible to reach the target population. According to literature, shorter distance to service delivery points and reduced waiting time improved health and nutrition outcomes of beneficiaries. Households with better water and sanitation facilities also better child nutrition indicators. The effect of war and insecurity on nutrition has been extensively explored. Scholars found that people born or living in war ravaged areas were less nourished (had lower dietary diversity scores) and had poorer nutritional indicators. Aside from infrastructure and insecurity, the capacity of program staff or volunteers to deliver an effective nutrition response also plays a critical role for success. Where capacity is limiting; coverage, impact and sustainability of nutrition programmes has failed resulting into increases in malnourished population and heightened the burden on health care systems [18-26]. Other challenges operational challenges included nomadic or mobile communities - which affected targeting and limited funding.

\section{b) Programmatic challenges}

These encompassed challenges related to the strategy and approach of nutrition response. They include preexisting conditions, practices and technicalities in delivery of programs 


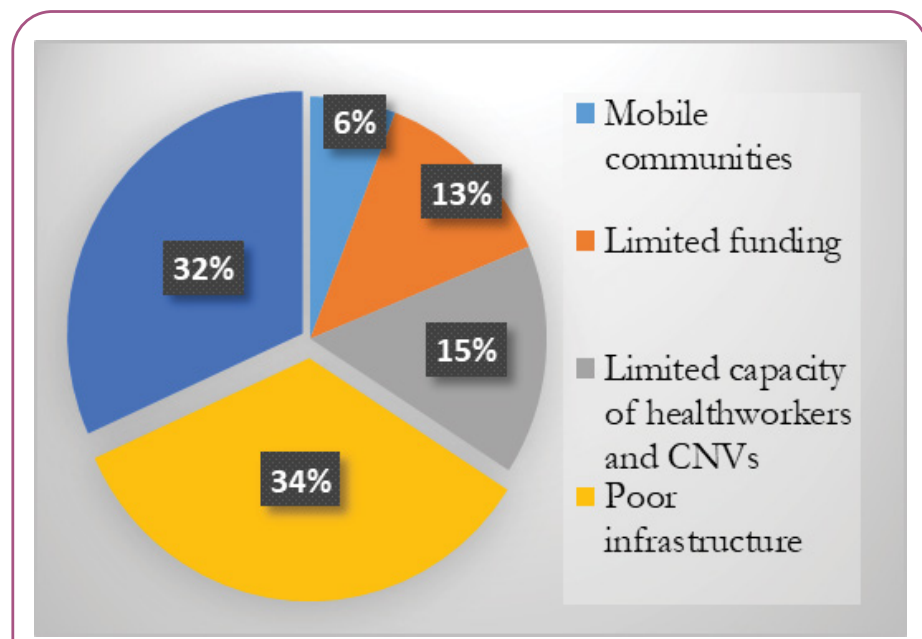

Figure 2 Operational challenges in implementing the nutrition specific programmes.

that in one way or another affected delivery, adherence and ultimately effectiveness and efficacy of interventions.

Figure 2 shows that of the commonly identified programmatic challenges were misuse of the therapeutic/supplementary food (37.5\%), pipeline breaks (15.6\%), negative cultural practices by mothers/caregivers (12.5\%) and default in participation by target beneficiaries. Seasonality age of care givers, number of relapses, non-response rate and alcoholism though important- were the least commonly ranked. All these either independently or in combination affected program success.

In line with daily dietary requirements, dosages of RUTFs are administered based on condition and weight of the beneficiary. Ideally, the recommended amounts should result into recovery and weight gain. Unfortunately, NGOs reported cases where the ration for an individual was shared between siblings or not administered as per protocol. Although the amount of RUTFs can be altered as nutrition condition improves, under dosage of RUTFs in acutely malnourished persons may render the approach ineffective for management of SAM especially among children below one year. The efficacy of RUTFs also depends on how early malnutrition is detected and responded to $[27,28]$.

Moreover, studies have shown that alternatives to standard RUTFs were not as effective [29]. This means that where the dosage of RTUFs was reduced by sharing, default in participation or lapses on administration by care givers, gains in nutritional outcomes were likely to be lower.

Analysis also shows that success of nutrition specific programs varied buy season. Although GAM rates have remained below critical levels of $15 \%$ (WHO threshold) with a peak registered between March-July 2017 (during the hunger gap). The trends show short term seasonal variations in GAM. This suggests despite factors like poor infrastructure, insecurity, limited capacity of health workers and CNVs to manage acute malnutrition and misuse of therapeutic foods, seasonality might have a bearing on malnutrition. Seasonality is closely linked to agricultural season and disease occurrence, which together have significant impact on household food and nutrition security.

\section{Lessons from implementing the nutrition specific programmes}

When asked to share insights on what they have learnt from implementing nutrition programs over the years, the key informants identified three critical aspects: community involvement, integrating approaches, and government ownership.

Table 3 provides a summary of the lessons that have been drawn by the different NGOs in the nutrition response.

\section{Community involvement}

The role of community participation for program success is not new. The ability of interventions to identify and appropriately respond to human development needs is intricately hinged to how the community has been infiltrated and involved. Where communities were involved, the acceptance and utilization of nutrition responses was noticeably higher.

\section{Integrated approaches and tackling underlying cause of Malnutrition}

Although informants hailed the current nutrition specific responses for being systematic, they argued that the programs were unintegrated. Examples were cited where facility-based treatment of malnutrition was provided without adequate water and sanitation facilities and nutrition education. According to UNICEF, there are a numerous determinant of malnutrition. Key among these are an individual's health status, access to health care, diverse nutritious foods, safe water, adequate sanitation and hygiene among others. Unfortunately, where one or a combination of any of these is defunct, the ability of the body to obtain and utilize food may be negatively affected. The lancet series of 2013 has elaborated the importance using a combination of nutrition specific and nutrition sensitive interventions and ensuring that nutrient intake is not emphasized at the expense of health and care practices [30-31].

\section{Involvement of Government}

At the moment the delivery of nutrition specific interventions in

Table 2 Characteristics of respondents.

\begin{tabular}{|c|c|}
\hline Characteristics & $n(\%)$ \\
\hline \multicolumn{2}{|c|}{ Designation } \\
\hline Program/Nutrition Manager & $5(28)$ \\
\hline Nutritionist & $10(56)$ \\
\hline Nurse/Midwife & $3(17)$ \\
\hline \multicolumn{2}{|c|}{ Years of Experience } \\
\hline 0-36 months & $7(39)$ \\
\hline $37-60$ months & $11(61)$ \\
\hline \multicolumn{2}{|c|}{ Education } \\
\hline Medical & $3(17)$ \\
\hline Public Health & $3(17)$ \\
\hline Food Science/ Nutrition & $11(61)$ \\
\hline Biochemistry & $1(6)$ \\
\hline
\end{tabular}


Table 3 Summary of lessons learnt from implementing nutrition programs.

\begin{tabular}{|c|c|}
\hline Rank & Description \\
\hline 1 & Programme is best implemented when the community is well consulted and engaged \\
\hline 2 & $\begin{array}{l}\text { The core components of CMAM alone did not reduce the prevalence of malnutrition except when root causes were addressed using } \\
\text { integrated approaches }\end{array}$ \\
\hline 3 & Nutrition outcomes only improved when government was involved \\
\hline 4 & Bad cultural beliefs and practices retarded performance \\
\hline 5 & Limited access to food and mobility of the communities decreased rate of program default \\
\hline 6 & Early detection for improved treatment outcomes \\
\hline 7 & Extending services closer to the population \\
\hline 8 & Use of locally existing structures in programme implementation \\
\hline \multirow[t]{2}{*}{9} & Collaboration among partners for referral linkage \\
\hline & Training and capacity building for all categories of staff \\
\hline
\end{tabular}

Eastern Equatoria state are broad in terms of how the intervention logic elicits use of the different protocols as prescribed by the WHO. The programs are undertaken at health facilities, within communities or both. However, dominance of NGOs tends to steer nutrition programming towards donor interest and specific donor requirements. There are limited efforts to coordinate actions and this tends to frustrate local ownership and sustainability. In most cases the duration of and mechanism of programming is tied to the presence of the donor and upon exit, the government is not prepared to continue or take on these programs. Respondents argued that where government was involved, nutritional outcomes were enhanced.

Broadly speaking, it is the role of governments to own the SDGs and develop guidelines/strategies to ensure achievement of all targets within their jurisdiction. In addition, governments also fund their national development priorities- including ensuring that their populations are adequately nourished. Studies show that governments who developed national guidelines for management of malnutrition, constructed and adequately equipped health facilities, trained both health professionals and community health workers and ensured continuous supply of medicines and therapeutic foods made significant strides in increasing access and coverage of nutritional programs as well as reduced the risk of nutrition related morbidity and mortality among children [35-37].

\section{Conclusions and Recommendations}

While global progress has been made in improving nutrition, malnutrition continues to be a complex problem especially in developing countries. It has negative consequences on health, physical and cognitive development, intellect and income. Optimal nutrition is therefore fundamental to achieving human development goals. For developing countries like South Sudan to achieve nutritional wellbeing amidst protracted conflict, interventions should holistically address all the root causes of malnutrition stipulated in the UNICEF conceptual framework (i.e., immediate, underlying and basic factors associated with malnutrition) otherwise malnutrition will remain and nutrition specific interventions would continue. This study has also shown that although nutrition specific responses play a vital role is tackling malnutrition, they are not sustainable. Challenges were particularly linked to regular stock-outs and high dependence on donor funding. Introduction of local alternative for RUTFs constituted from locally grown foods could also reduce the over reliance on donors as such an approach is likely to be cheaper. However, given the reduced efficacy as shown in literature, such alternatives would be used in non-acute cases. It will also be critical to undertake research on the best combination of locally available treatment methods and the availability of adequate foods to support such an approach. The introduction of already tested treatment approaches in similar contexts elsewhere could also be introduced and tested especially if local conditions can support sustainability to such methods.

Addressing the underlying causes of malnutrition coupled more sustainable treatment approaches could improve the sustainability of nutrition programmes. For example, scaling up nutrition sensitive livelihoods interventions could address the underlying causes of malnutrition and reduce the funding need for nutrition specific programmes. Other nutrition sensitive initiatives can include targeted social protection programmes such as cash for work and unconditional cash transfers should be integrated as part of nutrition programming especially during lean seasons when food shortages are heightened. Such programmes could target mothers and caregivers of malnourished children during peak lean seasons. However, a well-designed social protection approach will be necessary to ensure targeting is well managed and such as programme does not create negative incentives for mothers and caregivers to enroll on the programme such as deliberately starving their children.

In addition to locally tailored treatment methods, providing nutrition education to the community and community-based nutrition/health workers will enable spread of nutrition knowledge even when donors exit. Building the capacity of local community and health workers aids quick identifying of malnutrition and ensuring that they are treated. Community sensitization campaigns on the use of therapeutic or supplementary foods and the impact of such misuse on the treatment of malnutrition as well as campaigns against negative cultural practices could be helpful.

The fact that CMAM is a component of the Ministry of Health was perceived by some respondents as a positive aspect linked to sustainability. As such, capacity building of the government as a key partner for nutrition response remains critical for any successful implementation of nutrition programmes. Monitoring systems 
should not only be strengthened but should also adequately address questions related to the utilization of food, reasons for misuse of food and overall impact to so as to clearly inform the progress of nutrition programmes. Nutrition programmes should have proper beneficiary feedback mechanism to get feedback from beneficiaries regarding their concerns, issues lacking clarity and systems to address beneficiary queries and concerns coupled with sensitization which will probably reduce negative cultural practices

In addition to the challenges of poor infrastructure, limited funding and insecurity could be circumvented through investment particularly in health infrastructure and improving the quality of services. We conclude that in the short run, nutrition specific interventions can be effective in saving lives of acutely malnourished individuals among vulnerable populations.

\section{References}

1 Ozaltin M, Mas D, Strauss J (1997) The Nutrition is essential for a population and particularly children to survive grow and achieve their full potential in terms of social, economic, intellectual and physical milestones.

2 United Nations (2015) The United Nations adopted the Sustainable Development Goals (SDGs) - a set of 17 global goals with specific targets aimed at ending poverty, protecting the planet and ensuring prosperity for all by 2030 .

3 FAO, IFAD, UNICEF, WFP and WHO (2019) The State of Food Security and Nutrition in the World 2019. Safeguarding against economic slowdowns and downturns. Rome, FAO. Safeguarding against economic slowdowns and downturns. Rome, FAO.

4 IFPRI (2016) International Food Policy Research Institute (IFPRI): Global Food Policy Report 2016: pp 154.

5 WFP, IFAD (2012) The state of food insecurity in the world. Economic growth is necessary but not sufficient to accelerate reduction of hunger and malnutrition. Food and Agriculture Organization of the United Nations, Rome, Italy.

6 FAO, IFAD, UNICEF, WFP and WHO (2017) The State of Food Security and Nutrition in the World: Building resilience for peace and food security. Rome, FAO, Italy.

7 Black RE, Allen LH, Bhutta ZA (2008) Maternal and child under nutrition: Global and regional exposures and health consequences. Lancet 371: 243-260.

8 Black RE, Morris SS, Bryce J (2003) Where and why are 10 million children dying every year? Lancet 9376: 2226-2234.

9 http://www.ipcinfo.org/fileadmin/user_upload/ipcinfo/docs/IPC_ Alert_10_SouthSudan_Sept_2018.pd

10 South Sudan (2014-2018) Food Security and Nutrition Monitoring Bulletins: Analyses and assessments, Food security analysis (WFP-VAM).

11 Bain LE, Awah PK, Geraldine N, Kindong NP, Siga Y (2014) Malnutrition in Sub-Saharan Africa: Burden, causes and prospects. Pan African Medical Journal 15(1).

12 Bhutta ZA, Tahmeed A, Black RE (2008) What works? Interventions for maternal and child under nutrition and survival. The Lancet 9610: 417-440.
Currently, the nutrition specific nexus with food security, WASH, health, education and social-protection among others termed as 'nutrition sensitive interventions' desire a lot to admire but rather remains a daily humanitarian song without much success stories being documented [34]. The need to ensure both nutrition specific and nutrition-sensitive interventions are given equal investment priorities by allocating adequate resources while building communities resilience to local shocks is long overdue. A multi sectoral nutrition response is the way to go if we are to sustain the impact of treatment programs for malnutrition. In addition, Government, Non-Governmental Organizations and Donor community should concentrate on the promotion of economic, social and cultural rights and uses the experience of Food First Information and Action Network in the promotion of nutrition rights of the vulnerable population.

13 Bhutta ZA, Das JK, Rizvi A, Gaffey MF, Walker N (2013) Evidence-based interventions for improvement of maternal and child nutrition: what can be done and at what cost? The Lancet 9890: 452-477.

14 Aguayo VM, Badgaiyan N, Paintal K (2015) Determinants of child stunting in the Royal Kingdom of Bhutan: an in-depth analysis of nationally representative data. Maternal \& Child Nutrition 11 333-345.

15 Isanaka S, Villamor E, Shepherd S (2009) Assessing the impact of the introduction of the world health organization growth standards and weight-for-height z-score criterion on the response to treatment of severe acute malnutrition in children: Secondary data analysis. Pediatrics 2: 1.

16 WHO (2005) Report of an informal consultation on the communitybased management of severe malnutrition in children.

17 Ruel MT, Alderman H (2013) Maternal and child nutrition study group. Nutrition-sensitive interventions and programmes: How can they help to accelerate progress in improving maternal and child nutrition? The lancet 9891: 536-551.

18 Raveloharison A, Rakotonirina SC (2009) Landscape analysis on countries' readiness to accelerate action to reduce maternal and child undernutrition: The Madagascar assessment. SCN News: 43-48.

19 Ruel MT, Alderman H, Maternal \& Group C.N.S. (2013) Nutritionsensitive interventions and programmes: how can they help to accelerate progress in improving maternal and child nutrition? The Lancet 9891: 536-551.

20 Mavalankar D, Puthussery S, Menon K, Rana R, Bhandol J, et al. (2016) Effectiveness of nutrition interventions in low- and middleincome countries: An evidence summary. Protocol. London: EPPICentre, Social Science Research Unit, UCL Institute of Education, University College London, UK.

21 Olney DK, Pedehombga A, Ruel MT, Dillon A (2015) A 2-Year Integrated Agriculture and Nutrition and Health Behavior Change Communication Program Targeted to Women in Burkina Faso Reduces Anemia, Wasting, and Diarrhea in Children 3-12.9 Months of Age at Baseline: A Cluster-Randomized Controlled Trial.

22 Tapsoba S (2009) Towards Nutrition MDGs in Burkina Faso: Will capacity to act follow the commitment? SCN News: 23-30.

23 Dillon A, Bliznashka L, Olney D (2020) Experimental evidence on post-program effects and spillovers from an agriculture-nutrition program 36: 100820. 
24 Valdivia M (2004) Poverty, health infrastructure and the nutrition of Peruvian children. Economics \& Human Biology 2: 489-510.

25 Lee LF, Rosenzweig MR, Pitt MM (1997) The effects of improved nutrition, sanitation, and water quality on child health in highmortality populations. Journal of Econometrics 77: 209-236.

26 Dabalen AL, Paul S (2014) Effect of conflict on dietary diversity: Evidence from Côte d'Ivoire. World development 58: 143-158.

27 Fanzo JC, Graziose MM (2017) Developing capacity in nutrition. In nutrition and health in a developing world. Humana Press Cham pp 67-88).

28 Schoonees A, Lombard MJ, Musekiwa A, Nel E, Volmink J (2019) Ready-to-use therapeutic food (RUTF) for home-based nutritional rehabilitation of severe acute malnutrition in children from six months to five years of age. Cochrane Database of Systematic Reviews (5).

29 Kangas ST, Salpéteur C, Nikièma V, Talley L, Ritz C (2019) Impact of reduced dose of ready-to-use therapeutic foods in children with uncomplicated severe acute malnutrition: A randomized noninferiority trial in Burkina Faso. PLoS Medicine 16(8).

30 Kohlmann K, Callaghan-Gillespie M, Gauglitz JM, Steiner-Asiedu $M$, Saalia K (2019) Alternative ready-to-use therapeutic food yields less recovery than the standard for treating acute malnutrition in children from Ghana. Global Health: Science and Practice 7: 203-214

31 Gongwer CR, R Aryeetey (2014) Implementing nutrition interventions in Ghana at district level: Gaps and opportunities.

32 Deconinck H, Hallarou M, Criel B, Donnen P, Macq J (2016) Integrating acute malnutrition interventions into national health systems: lessons from Niger. BMC public health 16: 249.

33 United Nations Children's Fund (1998) The state of the world's children, 1998: Focus on nutrition. http://www.unicef.org/sowc98

34 Serdan GG (2009) The effects of the war in Iraq on nutrition and health: an analysis using anthropometric outcomes of children. Available at SSRN 1359161.

35 Narayan J, John D, Ramadas N (2019) Malnutrition in India: Status and government initiatives. Journal of public health policy 40: 126-141.

36 Saltzman A, Birol E, Bouis HE, Boy E, De Moura FF (2013) Biofortification: Progress toward a more nourishing future. Global Food Security 2: 9-17.

37 Shekar M, Ruel-Bergeron J, Herforth A (2013) Improving nutrition through multi-sectoral approaches. Washington, DC: International Bank for Reconstruction and Development, International Development Association of the World Bank. 